\title{
Estimativa da evapotranspiração de referência a partir de dados meteorológicos limitados
}

\author{
Daniel Fonseca de Carvalho(1), Hermes Soares da Rocha(2), Robson Bonomo ${ }^{(3)}$ e Adilson Pacheco de Souza(4) \\ (1)Universidade Federal Rural do Rio de Janeiro, Departamento de Engenharia, BR 465, Km 7, CEP 23897-000 Seropédica, RJ, Brasil. \\ E-mail: carvalho@ufrrj.br (2)Universidade de São Paulo, Escola Superior de Agricultura Luiz de Queiroz, Departamento de Engenharia \\ de Biossistemas, Avenida Pádua Dias, no 11, Caixa Postal 09, CEP 13418-900 Piracicaba, SP, Brasil. E-mail: hermessrocha@gmail.com \\ (3)Universidade Federal do Espírito Santo, Centro Universitário Norte do Espírito Santo, BR 101 Norte, Km 60, Bairro Litorâneo, CEP $29932-540$ \\ São Mateus, ES, Brasil. E-mail: robson.bonomo@ufes.br ${ }^{(4)}$ Universidade Federal do Mato Grosso, Instituto de Ciências Agrárias e Ambientais, \\ Campus de Sinop, Distrito Industrial, CEP 78550-000 Sinop, MT, Brasil. E-mail: adilsonpacheco@ufmt.br
}

Resumo - O objetivo deste trabalho foi avaliar a estimativa da evapotranspiração de referência (ETo), para a região Sudeste do Brasil, a partir de dados meteorológicos limitados. O método de Penman-Monteith FAO $56\left(\mathrm{PM}_{\mathrm{p}}\right)$ foi tomado como referência. Três cenários com dados meteorológicos limitados, obtidos de rede de estações automáticas, foram utilizados para estimação da ETo: método padrão $\left(\mathrm{PM}_{\mathrm{p}}\right)$ com uso da radiação solar estimada pelo balanço entre ondas curtas e longas $\left(\mathrm{PM}_{\mathrm{Kr}}\right)$; método padrão com uso da pressão de vapor estimada pelas temperaturas máxima e mínima, e pela umidade relativa do ar $\left(\mathrm{PM}_{\mathrm{ea}}\right)$; e método padrão com uso da velocidade de vento constante $\left(2 \mathrm{~m} \mathrm{~s}^{-1} ; \mathrm{PM}_{\mathrm{u} 2}\right)$. A ETo também foi estimada pelos métodos de Hargreaves-Samani (HS) e de Turc. Os modelos foram analisados por meio de indicadores estatísticos de desvio absoluto médio (MBE), erro relativo (ER), raiz quadrada do erro quadrático médio (RMSE) e índice de Willmott (d). O método $\mathrm{PM}_{\mathrm{ea}}$ é a melhor alternativa para estimar a ETo, seguido pelos métodos $\mathrm{PM}_{\mathrm{u} 2}$, para Espírito Santo e Rio de Janeiro, e PM $_{\mathrm{Kr} s}$, para São Paulo e Minas Gerais. Os maiores erros são obtidos com o método de Hargreaves-Samani, que superestimou a ETo em comparação ao $\mathrm{PM}_{\mathrm{p}}$, para a maioria das estações avaliadas.

Termos para indexação: Hargreaves-Samani, mínimo de dados, Penman-Monteith, radiação solar, Turc, velocidade do vento.

\section{Estimating reference evapotranspiration with limited meteorological data}

\begin{abstract}
The objective of this work was to evaluate the estimate of reference evapotranspiration (ETo) for the Southeast Region of Brazil using limited meteorological data. The FAO 56 Penman-Monteith method $\left(\mathrm{PM}_{\mathrm{p}}\right)$ was used as reference. Three scenarios of limited meteorological data, obtained from automatic network stations, were used for estimating ETo: standard method $\left(\mathrm{PM}_{\mathrm{p}}\right)$, with solar radiation estimated from the balance between short and wide wavelengths $\left(\mathrm{PM}_{\mathrm{Krs}}\right)$; standard method using vapor pressure estimated from minimum and maximum temperatures, and from air relative humidity $\left(\mathrm{PM}_{\mathrm{ea}}\right)$; and standard method using constant wind speed $\left(2 \mathrm{~m} \mathrm{~s}^{-1} ; \mathrm{PM}_{\mathrm{u} 2}\right)$. ETo was also estimated by the Hargreaves-Samani (HS) and Turc methods. The models were analyzed by statistical indicators of mean absolute deviation (MBE), relative error (RE), root mean square error (RMSE), and Willmott index (d). The $\mathrm{PM}_{\mathrm{ea}}$ method is the best alternative to estimate ETo, followed by $\mathrm{PM}_{\mathrm{u} 2}$, for the states of Espírito Santo and Rio de Janeiro, and PM $\mathrm{Kr}_{\mathrm{Kr}}$, for the states of São Paulo and Minas Gerais. The largest errors are obtained with the Hargreaves-Samani method, which overestimated ETo compared with $\mathrm{PM}_{\mathrm{p}}$, for most of the evaluated stations.
\end{abstract}

Index terms: Hargreaves-Samani, minimum data, Penman-Monteith, solar radiation, Turc, wind speed.

\section{Introdução}

A evapotranspiração (ET) é a variável mais ativa do ciclo hidrológico e a principal componente no balanço hídrico em ecossistemas agrícolas. Portanto, ela é um parâmetro chave para estudos de avaliação ambiental e de manejo de bacias hidrográficas (Raziei \& Pereira, 2013), para a estimativa da necessidade hídrica das culturas e para projetos e manejo de sistemas de irrigação (Kumar et al., 2008). As condições meteorológicas têm marcada influência sobre a ET; assim, pequenos erros em sua estimativa têm elevado impacto sobre o cálculo do balanço hidrológico de uma região (Allen et al., 2011b).

A evapotranspiração de referência (ETo) é medida por meio de técnicas e princípios físicos relativamente 
complexos (Allen et al., 2011a), e a forma direta mais precisa para sua estimativa é o balanço hídrico no solo com uso de lisímetros. No entanto, em virtude das limitações associadas ao método, o uso de modelos físico-matemáticos tem se tornado alternativa prática para a estimação da ETo. A partir de resultados de inúmeros estudos conduzidos nas últimas décadas, a equação combinada de Penman-Monteith (PM), modificada por Allen et al. (1998), é a que melhor representa os fatores físicos e fisiológicos que regem o processo da evapotranspiração. A principal desvantagem dessa equação é o grande número de variáveis meteorológicas necessárias para a sua aplicação, ao se considerar que muitas estações meteorológicas não têm todos os sensores necessários, os quais, mesmo quando presentes, muitas vezes, fornecem dados com baixa qualidade (Droogers \& Allen, 2002). Esse fato é importante, especialmente para países em desenvolvimento, onde conjuntos confiáveis de dados de radiação, umidade relativa do ar e velocidade do vento nem sempre são comuns (Trajkovic \& Kolakovic, 2009).

Vários métodos têm sido desenvolvidos e aplicados em práticas hidrológicas nas últimas décadas para preencher séries incompletas ou para suprir dados no caso de ausência de estações meteorológicas em determinadas regiões. Esses métodos baseiam-se em análises de regressão, análises de probabilidades, redes neurais artificiais (Landeras et al., 2008), programação genética e, também, no uso de equações de estimativa de variáveis meteorológicas com base física, como estimativas de radiação e umidade relativa com base na temperatura do ar (Allen et al., 1998). Essa técnica tem proporcionado estimativas aceitáveis de ETo pelo método de Penman-Monteith, com uso de dados mínimos (Martinez \& Thepadia, 2010; Sentelhas et al., 2010; Rocha et al., 2011), mas ainda é pouco utilizada no Brasil.

A abordagem de estimação da ETo a partir de dados mínimos ou limitados envolve a exploração de formas alternativas de cálculo, quando não se dispõe de série de dados meteorológicos com todos os parâmetros necessários para aplicação do método padrão da FAO $\left(\mathrm{PM}_{\mathrm{p}}\right)$. Como exemplo, podem-se citar a estimativa da radiação solar baseada na diferença entre as temperaturas máxima e mínima do ar e o fator associado à posição do local da estação em relação ao litoral $\left(\mathrm{PM}_{\mathrm{Krs}}\right)$, o cálculo da pressão atual de vapor com base nas temperaturas máxima e mínima e na umidade relativa do ar $\left(\mathrm{PM}_{\mathrm{ea}}\right)$, a utilização de velocidade do vento constante e igual a $2 \mathrm{~m} \mathrm{~s}^{-1}$ no modelo de Penman-Monteith $\left(\mathrm{PM}_{\mathrm{u} 2}\right)$, ou a aplicação dos modelos de Hargreaves-Samani (HS) e Turc.

O objetivo deste trabalho foi avaliar a estimativa da evapotranspiração de referência (ETo), para a região Sudeste do Brasil, a partir de dados meteorológicos limitados.

\section{Material e Métodos}

O trabalho foi desenvolvido com dados da região Sudeste do Brasil $\left(14^{\circ} 12^{\prime}\right.$ e $25^{\circ} 18^{\prime} \mathrm{S}, 39^{\circ} 40^{\prime}$ e $\left.53^{\circ} 10^{\prime} \mathrm{W}\right)$, que apresenta $35,6 \%$ da área irrigada do País e 9,1\% da área cultivada, e é responsável por 19,2 milhões de toneladas de cereais, leguminosas e oleaginosas, o que representa $10,5 \%$ da produção agrícola brasileira total (Instituto Brasileiro de Geografia e Estatística, 2013). As informações meteorológicas foram obtidas junto à rede de estações automáticas do Instituto Nacional de Meteorologia (Inmet), com registros no período de 1/1/2008 a 20/3/2011, com informação horária de temperatura e umidade relativa do ar, temperatura do ponto de orvalho $\left(\mathrm{T}_{\mathrm{po}}\right)$, pressão atmosférica, velocidade e direção do vento, radiação solar e precipitação pluvial.

De acordo com a disponibilidade dos dados, foram selecionadas oito estações no Rio de Janeiro, 26 em Minas Gerais, seis no Espírito Santo e seis em São Paulo (Tabela 1). Apesar de a série histórica ter sido pequena, a indisponibilidade de informações para estudos dessa natureza tem feito com que diversos deles sejam desenvolvidos com séries ainda menores (Douglas et al., 2009; Sentelhas et al., 2010).

Os dados de cada estação foram trabalhados para se obter os valores médios diários de todos os elementos meteorológicos. Em seguida, procedeu-se ao cálculo da ETo diária com uso do modelo Penman-Monteith FAO $56\left(\mathrm{PM}_{\mathrm{p}}\right)$, conforme Allen et al. (1998). Esse método foi utilizado como referência para avaliação dos demais modelos.

Três cenários com dados meteorológicos limitados foram utilizados para estimação da ETo: método padrão $\left(\mathrm{PM}_{\mathrm{p}}\right)$ com uso da radiação solar estimada pelo balanço entre ondas curtas e longas $\left(\mathrm{PM}_{\mathrm{Krs}}\right)$; método padrão com uso da pressão de vapor estimada pelas temperaturas máxima e mínima, e pela umidade relativa 
Tabela 1. Rede de estações meteorológicas automáticas do Instituto Nacional de Meteorologia utilizadas no estudo.

\begin{tabular}{|c|c|c|c|c|c|}
\hline Estação & Código & $\begin{array}{l}\text { Latitude } \\
\left({ }^{\circ} \mathrm{S}\right)\end{array}$ & $\begin{array}{l}\text { Longitude } \\
\left({ }^{\circ} \mathrm{W}\right)\end{array}$ & $\begin{array}{l}\text { Altitude } \\
\text { (m) }\end{array}$ & $\begin{array}{l}\text { Distância }^{(1)} \\
(\mathrm{km})\end{array}$ \\
\hline & \multicolumn{5}{|c|}{ Rio de Janeiro } \\
\hline Arraial do Cabo & A606 & 22,98 & 42,02 & 4 & 0,6 \\
\hline Campos dos Goytacazes & A607 & 21,71 & 41,34 & 25 & 32,3 \\
\hline Macaé & A608 & 22,38 & 41,81 & 32 & 5,5 \\
\hline Parati & A619 & 23,22 & 44,73 & 4 & 9,5 \\
\hline Petrópolis & A610 & 22,47 & 43,29 & 1.777 & 28,9 \\
\hline Resende & A609 & 22,45 & 44,44 & 452 & 64,4 \\
\hline Forte de Copacabana & A652 & 22,99 & 43,19 & 45 & 0,4 \\
\hline \multirow[t]{2}{*}{ Seropédica } & A601 & 22,76 & 43,86 & 34 & 22,7 \\
\hline & \multicolumn{5}{|c|}{ Minas Gerais } \\
\hline Aimorés & A534 & 19,50 & 41,06 & 193 & $>20$ \\
\hline Buritis & A544 & 15,52 & 46,44 & 894 & $>20$ \\
\hline Caldas & A530 & 21,92 & 46,38 & 1.150 & $>20$ \\
\hline Campina Verde & A519 & 19,53 & 49,53 & 547 & $>20$ \\
\hline Capelinha & A531 & 17,71 & 42,39 & 932 & $>20$ \\
\hline Caratinga & A554 & 19,74 & 42,14 & 615 & $>20$ \\
\hline Conceição das Alagoas & A520 & 19,99 & 48,15 & 568 & $>20$ \\
\hline Curvelo & A538 & 18,75 & 44,45 & 670 & $>20$ \\
\hline Diamantina & A537 & 18,23 & 43,65 & 1356 & $>20$ \\
\hline Espinosa & A543 & 14,92 & 42,80 & 570 & $>20$ \\
\hline Formiga & A524 & 20,46 & 45,45 & 878 & $>20$ \\
\hline Governador Valadares & A532 & 18,79 & 41,99 & 263 & $>20$ \\
\hline Itaobim & A550 & 16,34 & 41,29 & 266 & $>20$ \\
\hline Ituiutaba & A512 & 18,95 & 49,53 & 560 & $>20$ \\
\hline Juiz de Fora & A518 & 21,77 & 43,36 & 950 & $>20$ \\
\hline Mocambinho & A539 & 15,09 & 44,02 & 460 & $>20$ \\
\hline Muriaé & A517 & 21,11 & 42,38 & 270 & $>20$ \\
\hline Patrocínio & A523 & 19,00 & 46,99 & 963 & $>20$ \\
\hline Pirapora & A545 & 17,26 & 44,84 & 503 & $>20$ \\
\hline Salinas & A552 & 16,16 & 42,31 & 495 & $>20$ \\
\hline São João Del Rei & A514 & 21,11 & 44,25 & 991 & $>20$ \\
\hline Teófilo Otoni & A527 & 17,89 & 41,52 & 475 & $>20$ \\
\hline Três Marias & A528 & 18,20 & 45,46 & 921 & $>20$ \\
\hline Unaí & A542 & 16,55 & 46,88 & 631 & $>20$ \\
\hline Varginha & A515 & 21,57 & 45,40 & 925 & $>20$ \\
\hline \multirow[t]{2}{*}{ Viçosa } & A510 & 20,77 & 42,87 & 712 & $>20$ \\
\hline & \multicolumn{5}{|c|}{ Espírito Santo } \\
\hline Alegre & A617 & 20,75 & 41,49 & 138 & 79,1 \\
\hline Alfredo Chaves & A615 & 20,64 & 40,74 & 35 & 22,7 \\
\hline Linhares & A614 & 19,36 & 40,07 & 40 & 37,9 \\
\hline Santa Tereza & A613 & 19,99 & 40,58 & 998 & 41,3 \\
\hline São Mateus & A616 & 18,71 & 39,85 & 39 & 10,9 \\
\hline \multirow[t]{2}{*}{ Vitória } & A612 & 20,32 & 40,32 & 9 & 0,2 \\
\hline & \multicolumn{5}{|c|}{ São Paulo } \\
\hline Iguape & A712 & 24,67 & 47,55 & 3 & 4,0 \\
\hline Itapeva & A714 & 23,98 & 48,89 & 707 & $>20$ \\
\hline Ourinhos & A716 & 22,95 & 49,89 & 448 & $>20$ \\
\hline Presidente Prudente & A707 & 22,12 & 51,40 & 436 & $>20$ \\
\hline Sorocaba & A713 & 23,43 & 47,59 & 609 & $>20$ \\
\hline Valparaíso & A734 & 21,32 & 50,93 & 374 & $>20$ \\
\hline
\end{tabular}

(1)Distância da costa. do ar $\left(\mathrm{PM}_{\mathrm{ea}}\right)$; e método padrão com uso da velocidade de vento constante $\left(2 \mathrm{~m} \mathrm{~s}^{-1} ; \mathrm{PM}_{\mathrm{u} 2}\right)$. A ETo também foi estimada pelos métodos de Hargreaves-Samani (HS) e de Turc.

A equação de Allen et al. (1998), utilizada para o método $\mathrm{PM}_{\mathrm{p}}$, é descrita como:

$$
\text { ETo }=\frac{\left(0,408 \Delta(\mathrm{Rn}-\mathrm{G})+\gamma \frac{900}{\mathrm{~T}+273} \mathrm{u}_{2}\left(\mathrm{e}_{\mathrm{s}}-\mathrm{e}_{\mathrm{a}}\right)\right.}{\Delta+\gamma\left(1+0,34 \mathrm{u}_{2}\right)},
$$

em que: Rn é a radiação líquida na superfície da planta ( $\mathrm{MJ} \mathrm{m}^{-2}$ por dia); G é a densidade de fluxo de calor no solo ( $\mathrm{MJ} \mathrm{m}^{-2}$ por dia); $\mathrm{T}$ é a temperatura média do ar a $2 \mathrm{~m}$ de altura $\left({ }^{\circ} \mathrm{C}\right) ; \mathrm{u}_{2}$ é a velocidade do vento a $2 \mathrm{~m}$ de altura $\left(\mathrm{m} \mathrm{s}^{-1}\right)$; $\mathrm{e}_{\mathrm{s}}$ é a pressão de saturação de vapor $(\mathrm{kPa})$, obtida pela média entre $\mathrm{e}^{\circ}\left(\mathrm{T}_{\text {máx }}\right)$ e $\mathrm{e}^{\circ}\left(\mathrm{T}_{\text {mín }}\right) ; \mathrm{e}_{\mathrm{a}}$ é a pressão atual de vapor $(\mathrm{kPa}) ; \Delta$ é a declividade da curva de pressão de vapor $\left(\mathrm{kPa}^{\circ} \mathrm{C}^{-1}\right) ; \gamma$ é a constante psicrométrica $\left(\mathrm{kPa}^{\circ} \mathrm{C}^{-1}\right)$; e 0,408 é o fator de conversão para o termo $(\mathrm{Rn}-\mathrm{G})$, de $\mathrm{MJ} \mathrm{\textrm {m } ^ { - 2 }}$ por dia para milímetro por dia.

Para o método $\mathrm{PM}_{\mathrm{Kr}}$, a radiação solar (Rs) foi estimada pela seguinte equação:

$$
\mathrm{Rs}=\mathrm{K}_{\mathrm{Rs}} \sqrt{\left(\mathrm{T}_{\max }-\mathrm{T}_{\min }\right)} \mathrm{Ra}
$$

em que $\mathrm{K}_{\mathrm{Rs}}$ é o coeficiente de ajuste, que assume valor de $(0,16)^{\circ} \mathrm{C}^{-0,5}$ para áreas no interior do País (distância da costa maior que $20 \mathrm{~km})$, ou de $(0,19)^{\circ} \mathrm{C}^{-0,5}$ para áreas próximas ao litoral (Hargreaves \& Samani, 1985); $\mathrm{T}_{\text {máx }}$ é a temperatura máxima diária $\left({ }^{\circ} \mathrm{C}\right) ; \mathrm{T}_{\text {min }}$ é a temperatura mínima diária $\left({ }^{\circ} \mathrm{C}\right)$; e $\mathrm{Ra}$ é a radiação no topo da atmosfera (MJ m ${ }^{-2}$ por dia), calculada em função da latitude local e do dia do ano (Allen et al., 1998).

A expressão utilizada para estimar a pressão de vapor a partir das temperaturas mínima e máxima, e da umidade relativa foi a seguinte:

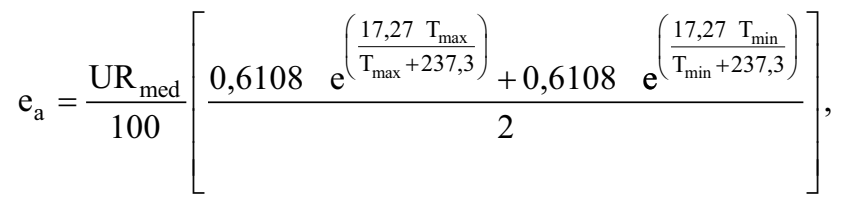

em que $\mathrm{UR}_{\text {med }}$ é a umidade relativa do ar média diária (\%).

As equações relativas aos métodos HS e de Turc são apresentadas a seguir: 


$$
\begin{gathered}
\text { ETo HS }=0,408\left[0,0023\left(\mathrm{~T}_{\text {med }}+17,8\right) \sqrt{\left(\mathrm{T}_{\max }-\mathrm{T}_{\min }\right)} \mathrm{Ra}\right] \mathrm{e} \\
\text { ETo Turc }=0,013 \frac{\mathrm{T}_{\text {med }}}{\mathrm{T}_{\text {med }}+15}(23,88 \mathrm{Rs}+50),
\end{gathered}
$$

em que $T_{\text {med }}$ é a temperatura média diária do ar.

Destaca-se que o método padronizado pela FAO preconiza o cálculo de $\mathrm{e}_{\mathrm{a}}$ a partir da $\mathrm{T}_{\mathrm{po}}$ (Carvalho \& Oliveira, 2012), e a Rs é medida ou estimada a partir dos coeficientes da equação de Angström-Prescott (Carvalho et al., 2011).

Pelo exposto, o cálculo da ETo com limitação de dados foi realizado com cinco metodologias alternativas $\left(\mathrm{ET}_{\mathrm{m}}\right)$, as quais foram comparadas ao método padrão de estimativa $\mathrm{PM}_{\mathrm{p}}\left(\mathrm{ET}_{\mathrm{p}}\right)$. Para a avaliação dos resultados, os valores de $\mathrm{ET}_{\mathrm{m}}$ foram comparados aos de $\mathrm{ET}_{\mathrm{p}}$ por meio dos índices estatísticos utilizados por Cai et al. (2007): coeficiente de determinação $\left(\mathrm{R}^{2}\right)$; coeficiente angular da regressão (b), obtido pela equação ajustada $\mathrm{de} \mathrm{ET}_{\mathrm{p}}$ e $\mathrm{ET}_{\mathrm{m}}$, ao passar pela origem; erro relativo (ER); erro absoluto médio (MBE); raiz do erro quadrático médio (RMSE); e índice de ajustamento de Willmott (d). Foram considerados satisfatórios os modelos que apresentaram $d \geq 0,95$ e $E R \leq 20 \%$, na distribuição de frequência acumulada do erro, bem como os modelos em que $\mathrm{b}$ assumia valores próximos a $1,0(1,0 \pm 0,10) \mathrm{e}$ $\mathrm{R}^{2} \geq 0,80$.

\section{Resultados e Discussão}

A variação anual dos valores de ETo, estimada pelo método $\mathrm{PM}_{\mathrm{p}}$ para estações meteorológicas localizadas em diferentes altitudes, nos quatro estados avaliados, foi semelhante ao comportamento sazonal da radiação global ao longo do ano, com maiores valores no verão e menores no inverno. De modo geral, se não há restrição hídrica, a evapotranspiração é proporcional à disponibilidade de energia solar e do saldo de radiação (Carvalho et al., 2011; Souza et al., 2011).

Nos estados litorâneos (Espírito Santo, Rio de Janeiro e São Paulo), as menores altitudes foram inferiores a $33 \mathrm{~m}$ (Tabela 1). Para Vitória, ES; Seropédica, RJ; e Iguape, SP, os resultados de ETo

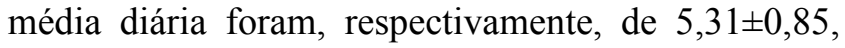
$4,87 \pm 1,12$ e 4,18 $\pm 0,69 \mathrm{~mm}$, no verão; $3,54 \pm 0,89$, $2,97 \pm 0,50$ e $2,40 \pm 0,71 \mathrm{~mm}$, no outono; $3,39 \pm 0,80$, $3,12 \pm 0,69$ e $1,97 \pm 0,56 \mathrm{~mm}$, no inverno; e 4,64 $\pm 0,90$, 4,34 $\pm 0,99$ e 3,56 $\pm 0,90 \mathrm{~mm}$, na primavera. Já os valores máximos anuais foram, respectivamente, de 7,28, 8,09 e 5,87 mm por dia, no verão, enquanto os mínimos anuais foram de 1,70 e $0,91 \mathrm{~mm}$ por dia, no inverno, para Vitória e Iguape, e de 1,40 mm por dia, no outono, para Seropédica.

Em Minas Gerais, estado não litorâneo, a estação com menor altitude (Aimorés) apresentou valores de ETo média, para as diferentes épocas do ano, de $5,45 \pm 0,79,3,26 \pm 0,81,3,43 \pm 0,97$ e $4,96 \pm 0,95 \mathrm{~mm}$ por dia no verão, no outono, no inverno e na primavera, respectivamente. De maneira geral, em Minas Gerais, os valores de ETo foram decrescentes de janeiro a junho e crescentes de julho a dezembro.

Em regiões litorâneas, a sazonalidade da ETo é influenciada pela variação da quantidade de vapor d'água na atmosfera local que, por sua vez, está relacionada à nebulosidade e sofre influência direta do balanço de ondas curtas e longas, com reflexos sobre o saldo de radiação. Assim, o desempenho da estimativa varia entre regiões úmidas, semiúmidas ou áridas, para um mesmo modelo (Souza et al., 2011).

As estimativas de ETo obtidas com os modelos alternativos diferiram das obtidas pelo método padrão $\mathrm{PM}_{\mathrm{p}}$, e o método $\mathrm{PM}_{\text {ea }}$ subestimou os valores de ETo, independentemente da estação meteorológica (Tabela 2). O método HS superestimou a ETo, na maioria das estações meteorológicas, e, portanto, não foi adequado às condições da região estudada, provavelmente pelo fato de o modelo ter sido desenvolvido em condições de clima árido (Hargreaves \& Samani, 1985). Apesar disso, Fernandes et al. (2012) ressaltam que melhores resultados podem ser obtidos com calibrações locais ou regionais.

Os coeficientes de regressão linear entre os métodos $\mathrm{PM}_{\mathrm{Krs}}$ e $\mathrm{PM}_{\mathrm{p}}$, e entre $\mathrm{PM}_{\mathrm{ea}}$ e $\mathrm{PM}_{\mathrm{p}}$ foram negativos e apresentaram valor residual negativo quando a ETo estimada por $\mathrm{PM}_{\mathrm{p}}$ tendeu a zero (Tabela 3). Isso indica que os métodos alternativos subestimaram os valores de ETo, independentemente da estação ou do estado avaliado.

As correlações obtidas entre os modelos HS e $\mathrm{PM}_{\mathrm{p}}$ foram as que apresentaram os menores coeficientes de determinação $\left(R^{2}\right)$, que variaram entre 0,4054 (Paraty, RJ) e 0,9119 (Caldas, MG). Observou-se que o método HS apresentou maior tendência a superestimar a ETo. Esse resultado diverge dos de Souza et al. (2011), que relataram subestimativa da ETo pelo método de HS, em condição de céu parcialmente coberto. Em condições de clima úmido, Trajkovic \& Kolakovic (2009) 
Tabela 2. Média \pm desvio-padrão das estimativas de evapotranspiração de referência (ETo), em milímetro por dia, obtida por diferentes modelos de estimativa da ETo, para estações meteorológicas da região Sudeste do Brasil(1).

\begin{tabular}{|c|c|c|c|c|c|c|c|c|}
\hline Estação & Média $\mathrm{PM}_{\mathrm{p}}$ & Mínima $\mathrm{PM}_{\mathrm{p}}$ & Máxima $\mathrm{PM}_{\mathrm{p}}$ & Média $\mathrm{PM}_{\mathrm{ea}}$ & Média $\mathrm{PM}_{\mathrm{Krs}}$ & Média HS & Média Turc & Média $\mathrm{PM}_{\mathrm{u} 2}$ \\
\hline & \multicolumn{8}{|c|}{ Espírito Santo } \\
\hline Alegre & $4,09 \pm 1,15$ & 1,73 & 6,82 & $3,96 \pm 1,14$ & $3,80 \pm 1,04$ & $4,36 \pm 1,15$ & $4,23 \pm 1,02$ & $4,54 \pm 1,18$ \\
\hline Alfredo Chaves & $4,00 \pm 1,15$ & 1,66 & 7,35 & $3,82 \pm 1,13$ & $3,83 \pm 1,05$ & $4,17 \pm 1,12$ & $3,91 \pm 0,95$ & $4,24 \pm 1,13$ \\
\hline Linhares & $4,29 \pm 1,10$ & 1,94 & 7,25 & $4,01 \pm 1,04$ & $3,85 \pm 0,94$ & $3,90 \pm 0,93$ & $4,11 \pm 0,97$ & $4,26 \pm 1,05$ \\
\hline São Mateus & $4,12 \pm 1,11$ & 1,90 & 6,82 & $3,90 \pm 1,09$ & $4,04 \pm 0,93$ & $3,91 \pm 0,88$ & $4,10 \pm 1,08$ & $4,14 \pm 1,08$ \\
\hline Santa Teresa & $3,29 \pm 0,99$ & 0,92 & 6,02 & $3,09 \pm 0,96$ & $2,92 \pm 0,84$ & $3,28 \pm 0,94$ & $3,54 \pm 0,94$ & $3,33 \pm 1,00$ \\
\hline \multirow[t]{2}{*}{ Vitória } & $4,23 \pm 1,18$ & 1,70 & 7,28 & $4,07 \pm 1,16$ & $4,08 \pm 1,06$ & $4,01 \pm 0,94$ & $4,31 \pm 1,06$ & $4,42 \pm 1,11$ \\
\hline & \multicolumn{8}{|c|}{ Rio de Janeiro } \\
\hline Arraial do cabo & $4,17 \pm 1,46$ & 0,28 & 8,28 & $3,90 \pm 1,15$ & $3,41 \pm 1,21$ & $2,67 \pm 0,69$ & $4,16 \pm 1,09$ & $3,97 \pm 1,29$ \\
\hline Campos dos Goytacazes & $4,31 \pm 1,23$ & 1,72 & 7,56 & $3,96 \pm 1,18$ & $3,94 \pm 1,05$ & $3,94 \pm 0,99$ & $4,04 \pm 1,00$ & $4,17 \pm 1,12$ \\
\hline Forte de Copacabana & $3,49 \pm 1,06$ & 1,41 & 6,96 & $3,31 \pm 1,04$ & $3,60 \pm 0,89$ & $3,27 \pm 0,96$ & $3,28 \pm 1,19$ & $3,47 \pm 1,07$ \\
\hline Macaé & $3,99 \pm 1,19$ & 1,64 & 6,81 & $3,69 \pm 1,11$ & $4,06 \pm 1,13$ & $3,90 \pm 0,98$ & $3,94 \pm 1,03$ & $4,01 \pm 1,09$ \\
\hline Paraty & $2,56 \pm 0,64$ & 1,32 & 4,50 & $2,42 \pm 0,65$ & $3,53 \pm 1,02$ & $3,74 \pm 0,99$ & $2,58 \pm 0,72$ & $2,87 \pm 0,64$ \\
\hline Petrópolis & $2,69 \pm 0,82$ & 0,58 & 5,35 & $2,43 \pm 0,75$ & $2,52 \pm 0,73$ & $2,58 \pm 0,76$ & $2,75 \pm 0,75$ & $2,62 \pm 0,77$ \\
\hline Resende & $3,58 \pm 1,01$ & 1,60 & 6,45 & $3,34 \pm 0,97$ & $3,60 \pm 1,00$ & $4,03 \pm 1,09$ & $3,57 \pm 0,88$ & $3,83 \pm 1,00$ \\
\hline \multirow[t]{2}{*}{ Seropédica } & $3,86 \pm 1,19$ & 1,40 & 8,09 & $3,60 \pm 1,15$ & $3,66 \pm 1,03$ & $4,09 \pm 1,11$ & $3,90 \pm 1,05$ & $3,98 \pm 1,17$ \\
\hline & \multicolumn{8}{|c|}{ São Paulo } \\
\hline Araçatuba & $4,57 \pm 1,09$ & 2,21 & 7,68 & $4,32 \pm 1,09$ & $4,53 \pm 1,01$ & $4,63 \pm 1,01$ & $4,03 \pm 0,94$ & $4,61 \pm 0,98$ \\
\hline Iguape & $3,03 \pm 1,16$ & 0,91 & 5,87 & $2,95 \pm 1,14$ & $3,14 \pm 1,12$ & $3,36 \pm 1,08$ & $3,37 \pm 1,11$ & $3,19 \pm 1,14$ \\
\hline Itapeva & $3,72 \pm 1,19$ & 1,54 & 6,77 & $3,44 \pm 1,13$ & $3,55 \pm 1,08$ & $3,67 \pm 1,13$ & $3,58 \pm 1,05$ & $3,73 \pm 1,14$ \\
\hline Ourinhos & $3,74 \pm 1,12$ & 1,58 & 6,25 & $3,60 \pm 1,12$ & $3,67 \pm 1,05$ & $4,27 \pm 1,13$ & $3,87 \pm 1,01$ & $4,19 \pm 1,11$ \\
\hline Presidente Prudente & $4,08 \pm 1,12$ & 1,88 & 6,92 & $3,93 \pm 1,12$ & $3,94 \pm 0,97$ & $4,21 \pm 1,09$ & $3,88 \pm 1,11$ & $4,51 \pm 1,08$ \\
\hline \multirow[t]{2}{*}{ Sorocaba } & $3,73 \pm 1,00$ & 1,60 & 6,08 & $3,48 \pm 0,98$ & $3,71 \pm 0,98$ & $4,05 \pm 1,07$ & $3,65 \pm 0,87$ & $3,88 \pm 0,99$ \\
\hline & \multicolumn{8}{|c|}{ Minas Gerais } \\
\hline Aimorés & $4,29 \pm 1,31$ & 1,80 & 8,44 & $4,20 \pm 1,24$ & $3,83 \pm 1,08$ & $4,40 \pm 0,92$ & $4,46 \pm 1,08$ & $4,87 \pm 1,07$ \\
\hline Buritis & $4,48 \pm 0,86$ & 2,11 & 7,09 & $4,29 \pm 0,84$ & $4,21 \pm 0,82$ & $4,17 \pm 0,72$ & $4,14 \pm 0,61$ & $4,50 \pm 0,79$ \\
\hline Caldas & $3,02 \pm 0,77$ & 1,40 & 5,10 & $2,91 \pm 0,76$ & $3,30 \pm 0,83$ & $3,97 \pm 0,84$ & $3,15 \pm 0,68$ & $3,43 \pm 0,73$ \\
\hline Campina Verde & $4,38 \pm 0,92$ & 2,20 & 7,06 & $4,23 \pm 0,90$ & $4,09 \pm 0,83$ & $4,48 \pm 0,80$ & $4,36 \pm 0,74$ & $4,52 \pm 0,76$ \\
\hline Capelinha & $3,65 \pm 0,89$ & 1,76 & 5,76 & $3,41 \pm 0,86$ & $3,72 \pm 0,90$ & $4,07 \pm 0,92$ & $3,53 \pm 0,77$ & $3,82 \pm 0,87$ \\
\hline Caratinga & $3,89 \pm 1,07$ & 1,88 & 6,90 & $3,79 \pm 1,06$ & $3,56 \pm 0,87$ & $4,09 \pm 0,88$ & $4,07 \pm 0,98$ & $4,27 \pm 1,03$ \\
\hline Curvelo & $4,45 \pm 1,00$ & 2,44 & 7,08 & $4,28 \pm 0,98$ & $4,16 \pm 0,88$ & $4,59 \pm 0,85$ & $4,43 \pm 0,80$ & $4,80 \pm 0,92$ \\
\hline Diamantina & $3,92 \pm 0,85$ & 1,62 & 5,99 & $3,64 \pm 0,82$ & $3,54 \pm 0,75$ & $3,47 \pm 0,76$ & $3,78 \pm 0,74$ & $3,85 \pm 0,82$ \\
\hline Espinosa & $5,28 \pm 0,85$ & 3,31 & 8,44 & $5,09 \pm 1,06$ & $4,95 \pm 0,95$ & $4,75 \pm 0,79$ & $4,61 \pm 0,82$ & $5,34 \pm 0,92$ \\
\hline Formiga & $3,80 \pm 0,89$ & 1,91 & 5,73 & $3,65 \pm 0,86$ & $3,77 \pm 0,85$ & $4,17 \pm 0,82$ & $3,75 \pm 0,70$ & $4,11 \pm 0,80$ \\
\hline Governador Valadares & $3,70 \pm 1,04$ & 1,74 & 6,07 & $3,52 \pm 1,04$ & $4,03 \pm 0,91$ & $4,56 \pm 0,95$ & $3,42 \pm 1,05$ & $4,17 \pm 0,99$ \\
\hline Itaobim & $4,77 \pm 1,30$ & 2,20 & 7,80 & $4,61 \pm 1,28$ & $4,52 \pm 1,02$ & $4,84 \pm 0,99$ & $4,48 \pm 1,18$ & $5,22 \pm 1,22$ \\
\hline Ituiutaba & $4,01 \pm 0,85$ & 2,26 & 6,16 & $3,85 \pm 0,83$ & $4,18 \pm 0,86$ & $4,79 \pm 0,83$ & $3,89 \pm 0,67$ & $4,58 \pm 0,78$ \\
\hline Juiz de Fora & $3,32 \pm 0,89$ & 1,42 & 5,65 & $3,07 \pm 0,86$ & $3,24 \pm 0,85$ & $3,41 \pm 0,94$ & $3,28 \pm 0,77$ & $3,34 \pm 0,88$ \\
\hline Mocambinho & $4,34 \pm 0,78$ & 2,86 & 6,33 & $4,26 \pm 0,79$ & $4,28 \pm 0,74$ & $5,07 \pm 0,83$ & $4,40 \pm 0,69$ & $5,13 \pm 0,84$ \\
\hline Muriaé & $3,84 \pm 1,07$ & 1,68 & 6,81 & $3,68 \pm 1,05$ & $3,69 \pm 1,00$ & $4,24 \pm 1,07$ & $3,93 \pm 0,93$ & $4,23 \pm 1,07$ \\
\hline Patrocínio & $3,77 \pm 0,81$ & 1,68 & 5,57 & $3,58 \pm 0,77$ & $3,89 \pm 0,85$ & $4,39 \pm 0,83$ & $3,72 \pm 0,63$ & $4,15 \pm 0,75$ \\
\hline Pirapora & $4,31 \pm 0,92$ & 2,64 & 6,55 & $4,19 \pm 0,92$ & $4,19 \pm 0,84$ & $4,76 \pm 0,79$ & $4,28 \pm 0,74$ & $4,89 \pm 0,87$ \\
\hline Salinas & $4,26 \pm 1,04$ & 2,24 & 6,53 & $4,19 \pm 1,03$ & $4,12 \pm 0,96$ & $4,85 \pm 0,96$ & $4,38 \pm 0,91$ & $4,97 \pm 1,03$ \\
\hline São João Del Rei & $3,71 \pm 0,87$ & 1,92 & 5,66 & $3,39 \pm 0,85$ & $3,84 \pm 0,85$ & $4,07 \pm 0,90$ & $3,44 \pm 0,76$ & $3,80 \pm 0,84$ \\
\hline Teófilo Otoni & $3,96 \pm 1,16$ & 1,98 & 6,84 & $3,83 \pm 1,12$ & $3,67 \pm 0,97$ & $4,08 \pm 0,93$ & $4,00 \pm 1,01$ & $4,32 \pm 1,08$ \\
\hline Três Marias & $4,40 \pm 0,89$ & 2,41 & 6,28 & $4,20 \pm 0,86$ & $4,06 \pm 0,83$ & $4,03 \pm 0,82$ & $4,14 \pm 0,69$ & $4,44 \pm 0,84$ \\
\hline Uberaba & $4,43 \pm 1,02$ & 2,06 & 7,27 & $4,15 \pm 0,96$ & $4,38 \pm 1,01$ & $4,63 \pm 0,91$ & $4,12 \pm 0,76$ & $4,61 \pm 0,90$ \\
\hline Unaí & $4,37 \pm 0,82$ & 2,24 & 6,20 & $4,22 \pm 0,81$ & $4,31 \pm 0,80$ & $4,77 \pm 0,77$ & $4,23 \pm 0,65$ & $4,80 \pm 0,82$ \\
\hline Varginha & $3,68 \pm 0,85$ & 1,85 & 5,84 & $3,51 \pm 0,82$ & $3,60 \pm 0,87$ & $3,99 \pm 0,90$ & $3,72 \pm 0,70$ & $3,96 \pm 0,82$ \\
\hline Viçosa & $3,17 \pm 0,95$ & 1,37 & 5,70 & $3,11 \pm 0,93$ & $3,23 \pm 0,87$ & $3,92 \pm 0,90$ & $3,43 \pm 0,86$ & $3,66 \pm 0,93$ \\
\hline
\end{tabular}

${ }^{(1)} \mathrm{PM}_{\mathrm{p}}$, método padrão, Penman-Monteith FAO 56; $\mathrm{PM}_{\mathrm{ea}}$, método padrão, com uso da pressão de vapor estimada pelas temperaturas máxima e mínima, e pela umidade relativa do ar; $\mathrm{PM}_{\mathrm{Kr}}$, método padrão, com uso da radiação solar estimada pelo balanço entre ondas curtas e longas; HS, método de HargreavesSamani; e $\mathrm{PM}_{\mathrm{u} 2}$, método padrão, com uso da velocidade de vento constante $\left(2,0 \mathrm{~m} \mathrm{~s}^{-1}\right)$. 
Tabela 3. Regressão linear e coeficiente de correlação para as dispersões entre a evapotranspiração de referência (EVo), estimada pelos modelos simplificados $\mathrm{PM}_{\mathrm{ea}}, \mathrm{PM}_{\mathrm{Kr}}$, HS, Turc e $\mathrm{PM}_{\mathrm{u} 2}(\mathrm{x})$, e o método padrão $\mathrm{PM}_{\mathrm{p}}(\mathrm{y})^{(1)}$.

\begin{tabular}{|c|c|c|c|c|c|c|c|c|c|c|c|c|c|c|c|}
\hline \multirow[t]{2}{*}{ Estação } & \multicolumn{3}{|c|}{$\mathrm{PM}_{\mathrm{p}} \mathrm{xPM}$ ea } & \multicolumn{3}{|c|}{$\mathrm{PM}_{\mathrm{p}} \mathrm{xPM_{ \textrm {Krs } }}$} & \multicolumn{3}{|c|}{$\mathrm{PM}_{\mathrm{p}} \mathrm{xHS}$} & \multicolumn{3}{|c|}{$\mathrm{PM}_{\mathrm{p}} \mathrm{x}$ Turc } & \multicolumn{3}{|c|}{$\mathrm{PM}_{\mathrm{p}} \mathrm{xPM} \mathrm{M}_{\mathrm{u} 2}$} \\
\hline & $\mathrm{a}$ & $\mathrm{b}$ & $\mathrm{r}$ & $\mathrm{a}$ & $\mathrm{b}$ & $\mathrm{r}$ & $\mathrm{a}$ & $\mathrm{b}$ & $\mathrm{r}$ & $\mathrm{a}$ & $\mathrm{b}$ & $\mathrm{r}$ & $\mathrm{a}$ & $\mathrm{b}$ & $\mathrm{r}$ \\
\hline & \multicolumn{15}{|c|}{ Espírito Santo } \\
\hline Alegre & $-0,0969$ & 0,9914 & 0,9992 & 0,3582 & 0,8417 & 0,9364 & 0,5527 & 0,9314 & 0,9338 & 0,7416 & 0,8531 & 0,9600 & 0,3757 & 1,0171 & 0,9942 \\
\hline Alfredo Chaves & $-0,1144$ & 0,9844 & 0,9921 & 0,3562 & 0,8683 & 0,9477 & 0,5869 & 0,8959 & 0,9188 & 0,6996 & 0,8044 & 0,9685 & 0,2947 & 0,9864 & 0,9975 \\
\hline Linhares & $-0,0446$ & 0,9455 & 0,9972 & 0,5242 & 0,7756 & 0,9088 & 0,6466 & 0,7578 & 0,8920 & 0,6020 & 0,8168 & 0,9222 & 0,2007 & 0,9471 & 0,9894 \\
\hline São Mateus & $-0,1122$ & 0,9738 & 0,9985 & 0,7827 & 0,7915 & 0,9472 & 0,8598 & 0,7403 & 0,9367 & 0,2214 & 0,9421 & 0,9748 & 0,1746 & 0,9630 & 0,9955 \\
\hline Santa Tereza & $-0,1078$ & 0,9722 & 0,9970 & 0,3178 & 0,7919 & 0,9287 & 0,4333 & 0,8671 & 0,9074 & 0,4875 & 0,9288 & 0,9764 & 0,0190 & 1,0077 & 0,9980 \\
\hline \multirow[t]{2}{*}{ Vitória } & $-0,0733$ & 0,9794 & 0,9989 & 0,5622 & 0,8312 & 0,9308 & 0,9388 & 0,7253 & 0,9184 & 0,6900 & 0,8564 & 0,9605 & 0,4558 & 0,9375 & 0,9970 \\
\hline & \multicolumn{15}{|c|}{ Rio de Janeiro } \\
\hline Arraial do Cabo & 0,6671 & 0,7739 & 0,9818 & 0,1272 & 0,7876 & 0,9501 & 1,1633 & 0,3602 & 0,7556 & 1,4575 & 0,6476 & 0,8621 & 0,3406 & 0,8704 & 0,9815 \\
\hline Campos dos Goytacazes & $-0,1475$ & 0,9537 & 0,9957 & 0,3908 & 0,8240 & 0,9637 & 0,7257 & 0,7466 & 0,9320 & 0,7096 & 0,7736 & 0,9526 & 0,2970 & 0,8980 & 0,9901 \\
\hline Forte de Copacabana & $-0,0871$ & 0,9736 & 0,9933 & 1,2645 & 0,6710 & 0,8014 & 0,8987 & 0,6809 & 0,7488 & $-0,4351$ & 1,0642 & 0,9446 & 0,0093 & 0,9922 & 0,9841 \\
\hline Macaé & 0,0067 & 0,9223 & 0,9935 & 0,5143 & 0,8871 & 0,9346 & 0,9494 & 0,7399 & 0,9028 & 0,6942 & 0,8116 & 0,9433 & 0,3845 & 0,9071 & 0,9896 \\
\hline Paraty & $-0,1438$ & 0,9999 & 0,9952 & 0,9337 & 1,0140 & 0,6420 & 1,2229 & 0,9822 & 0,6367 & 0,0243 & 0,9967 & 0,8972 & 0,3437 & 0,9871 & 0,9872 \\
\hline Petrópolis & 0,0224 & 0,8955 & 0,9861 & 0,3173 & 0,8184 & 0,9260 & 0,8933 & 0,6274 & 0,8805 & 0,5297 & 0,8277 & 0,9141 & 0,1528 & 0,9184 & 0,9804 \\
\hline Resende & $-0,1067$ & 0,9636 & 0,9981 & 0,2352 & 0,9395 & 0,9415 & 0,4265 & 1,0088 & 0,9331 & 0,5768 & 0,8375 & 0,9575 & 0,3028 & 0,9860 & 0,9892 \\
\hline \multirow[t]{2}{*}{ Seropédica } & $-0,1186$ & 0,9639 & 0,9969 & 0,5431 & 0,8068 & 0,9320 & 0,8317 & 0,8457 & 0,9111 & 0,6413 & 0,8457 & 0,9569 & 0,2075 & 0,9778 & 0,9948 \\
\hline & \multicolumn{15}{|c|}{ São Paulo } \\
\hline Araçatuba & $-0,2076$ & 0,9920 & 0,9962 & 0,4610 & 0,8916 & 0,9634 & 1,0511 & 0,7829 & 0,8461 & 0,5990 & 0,7512 & 0,8721 & 0,7360 & 0,8478 & 0,9421 \\
\hline Iguape & $-0,0379$ & 0,9857 & 0,9992 & 0,3815 & 0,9112 & 0,9455 & 0,7127 & 0,8748 & 0,9387 & 0,5353 & 0,9340 & 0,9757 & 0,2243 & 0,9775 & 0,9937 \\
\hline Itapeva & $-0,0962$ & 0,9504 & 0,9966 & 0,2682 & 0,8816 & 0,9724 & 0,3456 & 0,8942 & 0,9390 & 0,3968 & 0,8573 & 0,9713 & 0,2074 & 0,9475 & 0,9835 \\
\hline Ourinhos & $-0,1399$ & 0,9983 & 0,9984 & 0,3259 & 0,8922 & 0,9596 & 0,7285 & 0,9466 & 0,9400 & 0,5929 & 0,8756 & 0,9738 & 0,5407 & 0,9742 & 0,9827 \\
\hline Presidente Prudente & $-0,1257$ & 0,9952 & 0,9980 & 0,6448 & 0,8086 & 0,9337 & 0,6747 & 0,8674 & 0,8964 & 0,0300 & 0,9447 & 0,9571 & 0,7006 & 0,9341 & 0,9766 \\
\hline \multirow[t]{2}{*}{ Sorocaba } & $-0,1637$ & 0,9769 & 0,9953 & 0,1722 & 0,9474 & 0,9606 & 0,3392 & 0,9937 & 0,9220 & 0,5377 & 0,8348 & 0,9546 & 0,2569 & 0,9720 & 0,9816 \\
\hline & \multicolumn{15}{|c|}{ Minas Gerais } \\
\hline Aimorés & 0,1373 & 0,9459 & 0,9970 & 0,5120 & 0,7732 & 0,9394 & 1,5743 & 0,6585 & 0,9495 & 1,1916 & 0,7620 & 0,9245 & 1,4765 & 0,7903 & 0,9693 \\
\hline Buritis & $-0,0705$ & 0,9724 & 0,9956 & 0,1769 & 0,9010 & 0,9453 & 1,1694 & 0,6710 & 0,7970 & 1,3080 & 0,6329 & 0,8927 & 0,4501 & 0,9045 & 0,9795 \\
\hline Caldas & $-0,0745$ & 0,9893 & 0,9987 & 0,1731 & 1,0359 & 0,9506 & 0,8006 & 1,0495 & 0,9549 & 0,5681 & 0,8567 & 0,9699 & 0,6308 & 0,9288 & 0,9681 \\
\hline Campina Verde & $-0,0519$ & 0,9770 & 0,9964 & 0,4496 & 0,8310 & 0,9234 & 1,2168 & 0,7456 & 0,8623 & 1,1695 & 0,7283 & 0,9030 & 1,6703 & 0,6516 & 0,7913 \\
\hline Capelinha & $-0,0722$ & 0,9544 & 0,9958 & 0,2302 & 0,9563 & 0,9543 & 0,5863 & 0,9545 & 0,9238 & 0,5683 & 0,8108 & 0,9462 & 0,3188 & 0,9587 & 0,9816 \\
\hline Caratinga & $-0,0675$ & 0,9905 & 0,9990 & 0,6384 & 0,7501 & 0,9276 & 1,1077 & 0,7659 & 0,9323 & 0,6595 & 0,8772 & 0,9545 & 0,5816 & 0,9478 & 0,9896 \\
\hline Curvelo & $-0,0651$ & 0,9780 & 0,9986 & 0,4204 & 0,8405 & 0,9577 & 1,0670 & 0,7918 & 0,9318 & 1,0304 & 0,7641 & 0,9495 & 0,8040 & 0,8980 & 0,9775 \\
\hline Diamantina & $-0,1456$ & 0,9672 & 0,9966 & 0,3219 & 0,8212 & 0,9278 & 0,4485 & 0,7722 & 0,8633 & 0,5592 & 0,8233 & 0,9408 & 0,0701 & 0,9664 & 0,9918 \\
\hline Espinosa & $-0,0518$ & 0,9721 & 0,9987 & 0,5155 & 0,8384 & 0,9584 & 1,7607 & 0,5654 & 0,7881 & 1,2092 & 0,6455 & 0,8584 & 1,1315 & 0,7972 & 0,9464 \\
\hline Formiga & $-0,0106$ & 0,9628 & 0,9978 & 0,2731 & 0,9196 & 0,9615 & 0,9664 & 0,8429 & 0,9184 & 0,9285 & 0,7426 & 0,9390 & 0,8178 & 0,8660 & 0,9669 \\
\hline Governador Valadares & $-0,1960$ & 1,0026 & 0,9986 & 1,0588 & 0,8025 & 0,9131 & 1,4205 & 0,8486 & 0,9310 & $-0,1086$ & 0,9537 & 0,9460 & 0,7008 & 0,9361 & 0,9860 \\
\hline Itaobim & $-0,1053$ & 0,9889 & 0,9990 & 0,9360 & 0,7515 & 0,9582 & 1,4847 & 0,7038 & 0,9221 & 0,3617 & 0,8648 & 0,9521 & 0,8087 & 0,9247 & 0,9875 \\
\hline Ituiutaba & $-0,0370$ & 0,9686 & 0,9946 & 0,3216 & 0,9618 & 0,9527 & 1,3264 & 0,8628 & 0,8838 & 1,1277 & 0,6886 & 0,8731 & 1,1056 & 0,8659 & 0,9443 \\
\hline Juiz de Fora & $-0,1170$ & 0,9614 & 0,9950 & 0,3093 & 0,8830 & 0,9179 & 0,4736 & 0,8847 & 0,8380 & 0,5556 & 0,8202 & 0,9393 & 0,0859 & 0,9803 & 0,9902 \\
\hline Mocambinho & $-0,1134$ & 1,0078 & 0,9981 & 0,4283 & 0,8882 & 0,9320 & 0,8183 & 0,9801 & 0,9214 & 0,7728 & 0,8367 & 0,9387 & 0,7271 & 1,0157 & 0,9437 \\
\hline Muriaé & $-0,0632$ & 0,9767 & 0,9971 & 0,3412 & 0,8743 & 0,9378 & 0,6739 & 0,9305 & 0,9349 & 0,7990 & 0,8171 & 0,9400 & 0,4929 & 0,9740 & 0,9790 \\
\hline Patrocínio & 0,0247 & 0,9441 & 0,9962 & 0,1667 & 0,9884 & 0,9441 & 0,9169 & 0,9219 & 0,9058 & 0,9999 & 0,7226 & 0,9641 & 0,8310 & 0,8803 & 0,9548 \\
\hline Pirapora & $-0,0767$ & 0,9893 & 0,9982 & 0,4948 & 0,8569 & 0,9448 & 1,4607 & 0,7657 & 0,8915 & 1,1321 & 0,7303 & 0,9087 & 1,0150 & 0,8987 & 0,9555 \\
\hline Salinas & $-0,0175$ & 0,9859 & 0,9979 & 0,4855 & 0,8516 & 0,9298 & 1,2639 & 0,8423 & 0,9144 & 0,9294 & 0,8098 & 0,9260 & 0,8751 & 0,9610 & 0,9725 \\
\hline São João Del Rei & $-0,2400$ & 0,9784 & 0,9961 & 0,3305 & 0,9444 & 0,9585 & 0,5202 & 0,9556 & 0,9224 & 0,3141 & 0,8412 & 0,9595 & 0,2678 & 0,9525 & 0,9777 \\
\hline Teófilo Otoni & 0,0123 & 0,9650 & 0,9986 & 0,5712 & 0,7824 & 0,9388 & 1,1322 & 0,7443 & 0,9276 & 0,7170 & 0,8292 & 0,9576 & 0,6691 & 0,9222 & 0,9895 \\
\hline Três Marias & $-0,0414$ & 0,9644 & 0,9962 & 0,2156 & 0,8736 & 0,9406 & 0,7239 & 0,7519 & 0,8208 & 1,0515 & 0,7028 & 0,9148 & 0,4254 & 0,9135 & 0,9761 \\
\hline Uberaba & $-0,0212$ & 0,9424 & 0,9957 & 0,2029 & 0,9430 & 0,9524 & 1,0549 & 0,8072 & 0,8976 & 1,2537 & 0,6464 & 0,8632 & 0,8008 & 0,8607 & 0,9773 \\
\hline Unaí & $-0,0778$ & 0,9820 & 0,9977 & 0,3112 & 0,9144 & 0,9450 & 1,0094 & 0,8589 & 0,9223 & 1,0713 & 0,7210 & 0,9160 & 0,5791 & 0,9650 & 0,9745 \\
\hline Varginha & $-0,0297$ & 0,9613 & 0,9976 & 0,0699 & 0,9608 & 0,9416 & 0,4157 & 0,9723 & 0,9249 & 0,9375 & 0,7555 & 0,9137 & 0,4846 & 0,9451 & 0,9788 \\
\hline Viçosa & $-0,0282$ & 0,9883 & 0,9990 & 0,5164 & 0,8548 & 0,9286 & 1,0952 & 0,8884 & 0,9333 & 0,6132 & 0,8857 & 0,9680 & 0,6028 & 0,9627 & 0,9822 \\
\hline
\end{tabular}

(1) $\mathrm{PM}_{\mathrm{p}}$, método padrão, Penman-Monteith FAO 56; $\mathrm{PM}_{\mathrm{ea}}$, método padrão, com uso da pressão de vapor estimada pelas temperaturas máxima e mínima, e pela umidade relativa do ar; $\mathrm{PM}_{\mathrm{Kr} \text { s, }}$, método padrão, com uso da radiação solar estimada pelo balanço entre ondas curtas e longas; HS, método de HargreavesSamani; e $\mathrm{PM}_{\mathrm{u} 2}$, método padrão, com uso da velocidade de vento constante $\left(2,0 \mathrm{~m} \mathrm{~s}^{-1}\right)$. 
constataram espalhamento nos valores de ETo. Já para o modelo de Turc, as correlações com o método padrão foram maiores, com $\mathrm{R}^{2}$ entre 0,7368 e 0,9534 . Para esse modelo, as menores correlações foram verificadas em estações no Estado do Rio de Janeiro.

Independentemente da região (estação) ou do estado avaliado, os modelos com adaptações para obtenção das variáveis meteorológicas necessárias para estimativa da ETo $\left(\mathrm{PM}_{\mathrm{Krs}}\right.$ e $\left.\mathrm{PM}_{\mathrm{ea}}\right)$ propiciam melhores resultados que as equações empíricas e parametrizadas (HS e Turc). Entretanto, esses modelos ainda demandam medidas da velocidade do vento, que podem limitar a sua utilização.

A análise dos desvios relativos médios (ER) apontou que a probabilidade de ocorrerem erros superiores a
$20 \%$ foi de $8,77,10,68,7,4$ e $1,1 \%$ para as metodologias $\mathrm{PM}_{\mathrm{ea}}, \mathrm{PM}_{\mathrm{Krs}}$, HS e Turc, respectivamente. Para um erro de $21 \%$, a probabilidade de ocorrência foi de apenas 0,05\%, na comparação entre $\mathrm{PM}_{\mathrm{u} 2}$ e $\mathrm{PM}_{\mathrm{p}}$, em Espírito Santo (Figura 1 A). Para o Rio de Janeiro, erros maiores que $20 \%$ ocorreram com frequências de 0,58 , $21,61,39,18,10,89$ e 2,23\%, para os métodos $\mathrm{PM}_{\text {ea, }}$ $\mathrm{PM}_{\mathrm{Krs}}$, HS, Turc e $\mathrm{PM}_{\mathrm{u} 2}$, respectivamente (Figura $1 \mathrm{~B}$ ). No Estado de São Paulo, o erro máximo verificado para $\mathrm{PM}_{\text {ea }}$ foi de $16,63 \%$, com probabilidade de ocorrência de $0,05 \%$ dos dados, e probabilidades de ocorrência de $3,79,19,82,12,19$ e 8,26 , para desvios superiores a $20 \%$, foram obtidas para os métodos $\mathrm{PM}_{\mathrm{Krs}}, \mathrm{HS}$, Turc e $\mathrm{PM}_{\mathrm{u} 2}$ (Figura $1 \mathrm{C}$ ). Em Minas Gerais, o erro máximo foi de $19,36 \%$, para $\mathrm{PM}_{\text {ea }}$, com probabilidade

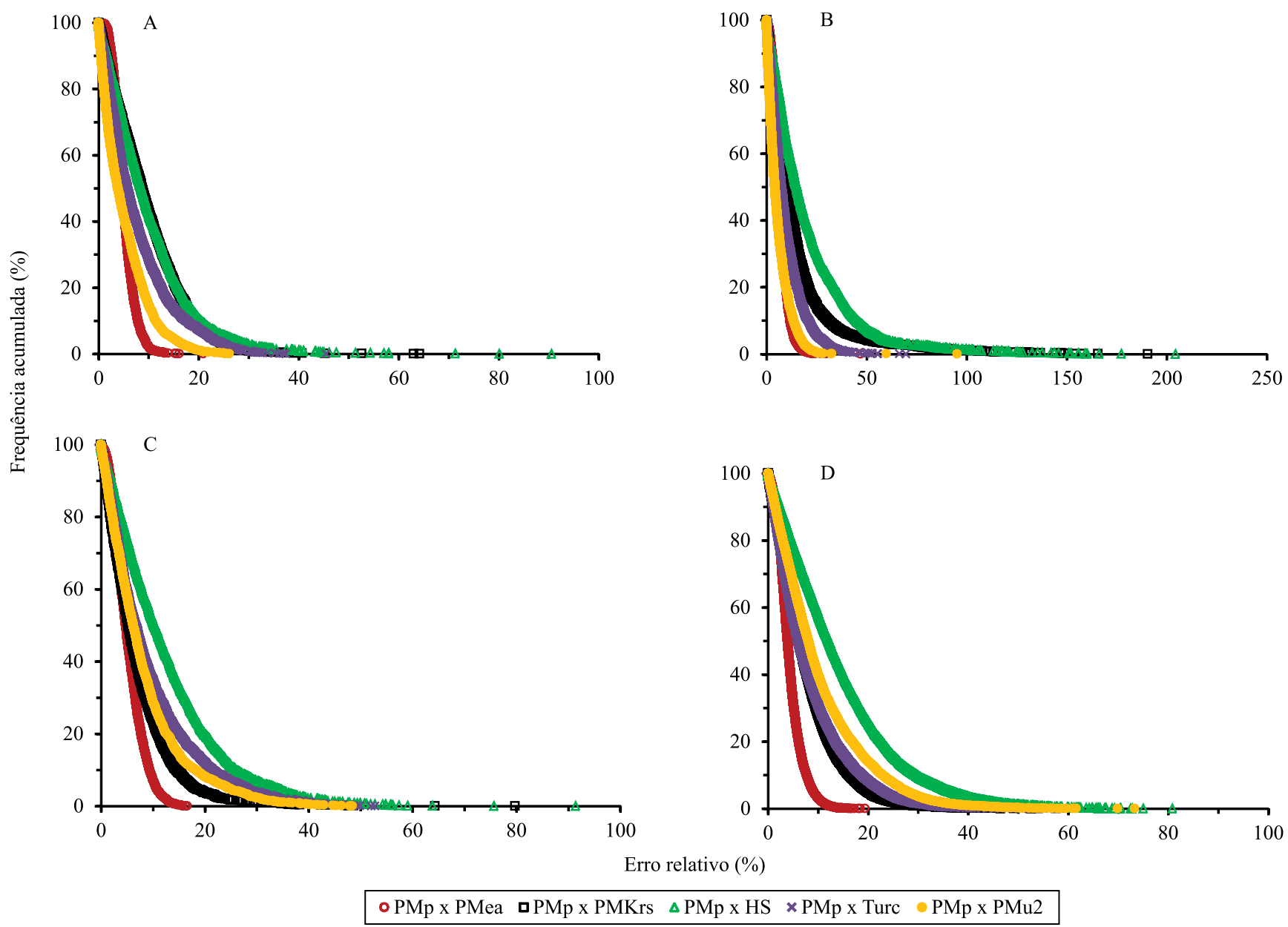

Figura 1. Frequência acumulada de ocorrência do erro relativo percentual para os modelos empíricos alternativos em relação ao método padrão da FAO $\left(\mathrm{PM}_{\mathrm{p}}\right)$, para estações dos Estados do Espírito Santo (A), do Rio de Janeiro (B), de São Paulo (C) e de Minas Gerais (D). $\mathrm{PM}_{\mathrm{ea}}$, método padrão, com uso da pressão de vapor estimada pelas temperaturas máxima e mínima, e pela umidade relativa do ar; $\mathrm{PM}_{\mathrm{Krs}}$, método padrão, com uso da radiação solar estimada pelo balanço entre ondas curtas e longas; HS, método de Hargreaves-Samani; e $\mathrm{PM}_{\mathrm{u} 2}$, método padrão, com uso da velocidade de vento constante $\left(2,0 \mathrm{~m} \mathrm{~s}^{-1}\right)$. 
de ocorrência de apenas $0,01 \%$; e a probabilidade de ocorrerem erros superiores a $20 \%$ foi de 4,94 , $25,93,8,37$ e $14,02 \%$, para $\mathrm{PM}_{\mathrm{Krs}}$, HS, Turc e $\mathrm{PM}_{\mathrm{u} 2}$, respectivamente (Figura $1 \mathrm{D}$ ).

Martí et al. (2010) consideram conveniente a utilização do indicador ER para inferir sobre a qualidade do desempenho de estimativas da ETo por diferentes modelos. Apesar de ocorrerem em baixa frequência, erros elevados foram registrados na comparação com o método $\mathrm{PM}_{\mathrm{p}}$, principalmente para o método HS no Rio de Janeiro, onde houve desvios de $204 \%$ em $0,03 \%$ dos dados. Os maiores erros encontrados com o método HS foram previstos, também, com a análise de correlação. Consequentemente, esse método não deve ser adotado na região.

Ao se considerar a distribuição de frequência dos desvios médios (Figura 1), o método $\mathrm{PM}_{\mathrm{ea}}$ consistiu na melhor alternativa para estimar a ETo, em todos os estados. Isso porque apresentou a menor ocorrência de erros elevados $\left(\mathrm{PM}_{\mathrm{p}} \times \mathrm{PM}_{\mathrm{ea}}\right)$, seguido dos métodos $\mathrm{PM}_{\mathrm{u} 2}$ $\left(\mathrm{PM}_{\mathrm{p}} \times \mathrm{PM}_{\mathrm{u} 2}\right)$, para Espírito Santo e Rio de Janeiro, e $\mathrm{PM}_{\mathrm{Krs}}\left(\mathrm{PM}_{\mathrm{p}} \times \mathrm{PM}_{\mathrm{Kr}}\right)$, para São Paulo e Minas Gerais.

De acordo com Souza et al. (2011), a utilização conjunta dos indicativos estatísticos MBE, RMSE e índice de ajustamento $\mathrm{d}$ de Willmott consiste numa avaliação adequada do desempenho estatístico de modelos de estimativa, com análises simultâneas dos desvios da média, para identificação da ocorrência de sub ou superestimativas, bem como para o espalhamento e o ajustamento dos modelos, em relação aos valores medidos. $\mathrm{O}$ indicativo MBE representa o desvio das médias e provê informações sobre o desempenho do modelo em longo prazo; valores negativos indicam subestimativa, e vice-versa. Quanto menor o valor absoluto de MBE, melhor é o desempenho do modelo testado (Stone, 1993); porém, uma superestimativa cancela uma subestimativa.

Nos Estados do Espírito Santo, do Rio de Janeiro e de São Paulo, o MBE não apresentou tendências significativas (Figura 2 A), com subestimativas máximas de $-1,51 \mathrm{~mm}$ por dia em Arraial do Cabo, $\mathrm{RJ}$, e superestimativas máximas de $0,53 \mathrm{~mm}$ por dia em Ourinhos, SP. O emprego da radiação solar global medida, com $e_{a}$ estimada em função da temperatura do ponto de orvalho e da velocidade do vento igual a $2 \mathrm{~m} \mathrm{~s}^{-1}$ $\left(\mathrm{PM}_{\mathrm{u} 2}\right)$, propiciou superestimativas da ETo em todas as estações avaliadas em Minas Gerais $(0,796 \mathrm{~mm}$ por dia para Mocambinho). Em geral, os menores valores absolutos de MBE foram gerados pelas adaptações de $\mathrm{PM}_{\mathrm{ea}}$ e $\mathrm{PM}_{\mathrm{Kr} s}$, indício de que medidas simplificadas e parametrizações permitem baixos desvios quanto aos valores médios, principalmente nas estações localizadas no Espírito Santo, no Rio de Janeiro e em São Paulo.

Rojas \& Sheffield (2013), em estudo no nordeste de Louisiana, EUA, constataram que o modelo de Turc subestimou a ETo padronizada em $10 \%$, enquanto o HS superestimou em $8 \%$, com valores de MBE de 0,6 e $0,83 \mathrm{~mm}$ por dia, e RMSE de 0,75 e $0,99 \mathrm{~mm}$ por dia, respectivamente. Os autores descrevem valores de 0,67 e $0,75 \mathrm{~mm}$ por dia para RMSE, ao considerarem a ETo estimada para velocidade média do vento na localidade e velocidade média global do vento, respectivamente.

$O$ indicador estatístico RMSE informa sobre o valor real do erro produzido pelo modelo e indica o espalhamento das estimativas da variável independente, para um determinado valor da variável dependente. Contudo, erros de grande proporção na soma podem causar acréscimos significativos nos valores de RMSE; além disso, o indicador não diferencia superestimativas de subestimativas. Quanto menores os valores obtidos para RMSE, melhor o desempenho dos modelos.

Os maiores espalhamentos gerados foram de 0,481 em Arraial do Cabo, RJ; 1,224 em Paraty, RJ; 1,827 em Arraial do Cabo, RJ; 0,884 em Espinosa, MG; e 0,843 $\mathrm{mm}$ por dia em Mocambinho, MG, para os modelos $\mathrm{PM}_{\mathrm{ea}}, \mathrm{PM}_{\mathrm{Krs}}, \mathrm{HS}$, Turc e $\mathrm{PM}_{\mathrm{u} 2}$, respectivamente. Em geral, os menores valores de RMSE foram obtidos pelos modelos $\mathrm{PM}_{\mathrm{ea}}$ e $\mathrm{PM}_{\mathrm{u} 2}$ (Figura $2 \mathrm{~B}$ ).

Todorovic et al. (2013) realizaram estimativas em 577 estações meteorológicas, distribuídas em 16 países do Mediterrâneo, e obtiveram desempenho semelhante para os modelos $\mathrm{HS}$ e $\mathrm{PM}_{\mathrm{u} 2}$, em regiões hiperáridas, com valores de RMSE em torno de $0,60-0,65 \mathrm{~mm}$ por dia. Segundo os autores, em regiões semiáridas ou úmidas, o método $\mathrm{PM}_{\mathrm{u} 2}$ apresentou melhores resultados, com o menor valor de RMSE, de $0,52 \mathrm{~mm}$ por dia. Os autores sugeriram, portanto, correções locais na temperatura do ponto de orvalho e ajustes no coeficiente empírico Krs, para melhorar o desempenho nas estimativas.

No presente trabalho, os valores de RMSE permaneceram na faixa de $0,05-0,85 \mathrm{~mm}$ por dia, com maiores RMSE para estações no interior do País. Esse resultado foi oposto ao observado por Martinez $\&$ Thepadia (2010), que relataram valores entre 0,40 e 1,23 $\mathrm{mm}$ por dia, para estações da Flórida, com média de $0,695 \mathrm{~mm}$ por dia. 

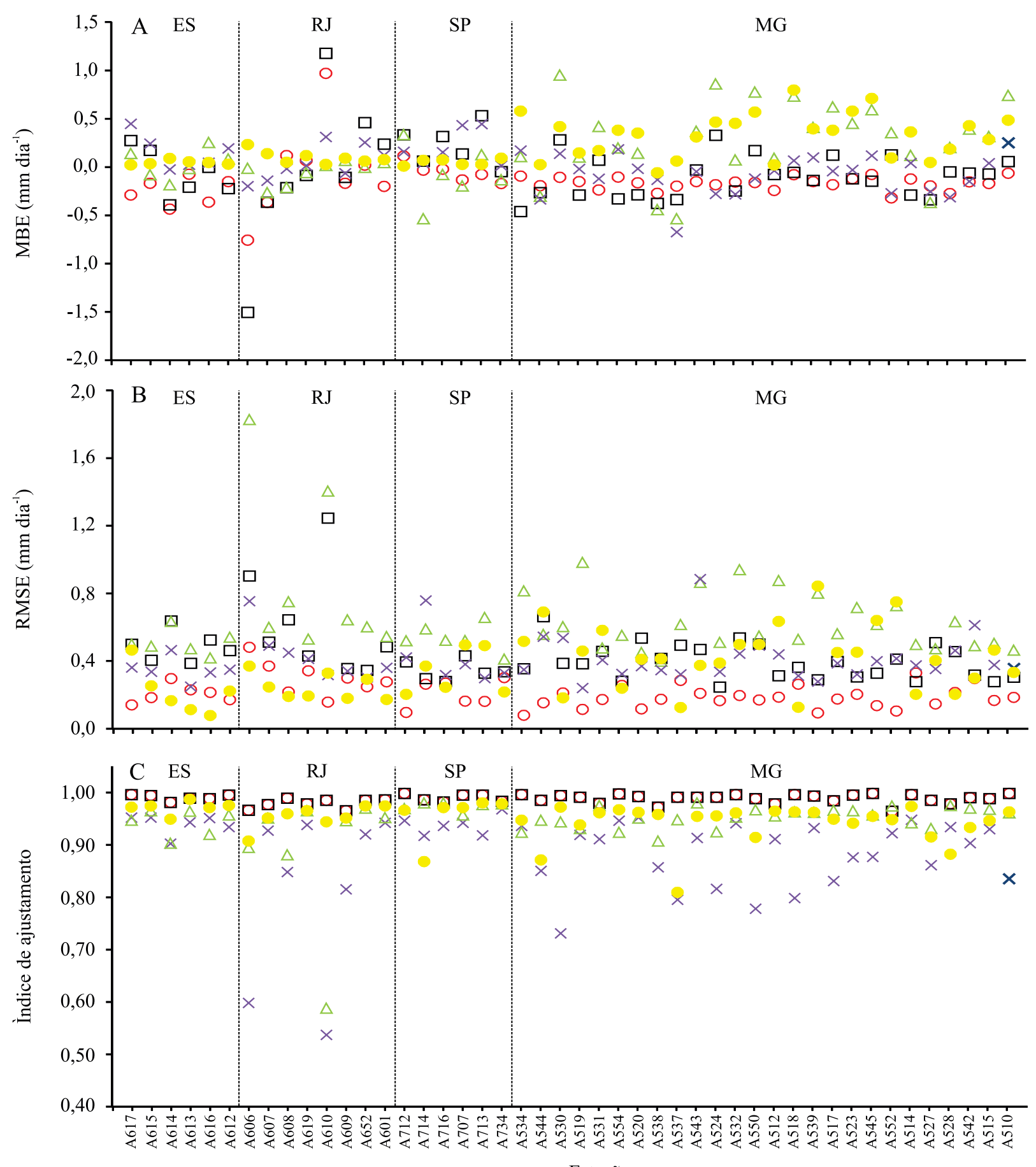

Estação

\begin{tabular}{|c|c|c|}
\hline OPMea & 口PMkrs & $\triangle \mathrm{HS}$ \\
\hline
\end{tabular}

Figura 2. Erro médio absoluto (MBE, A), raiz quadrada do erro quadrático médio (RMSE, B) e índice de ajustamento d de Willmott $(\mathrm{C})$ para evapotranspiração de referência diária ( $\mathrm{mm}$ por dia), calculada a partir de modelos empíricos alternativos em relação ao método padrão da $\mathrm{FAO}\left(\mathrm{PM}_{\mathrm{p}}\right)$. $\mathrm{PM}_{\mathrm{ea}}$, método padrão, com uso da pressão de vapor estimada pelas temperaturas máxima e mínima, e pela umidade relativa do ar; $\mathrm{PM}_{\mathrm{Kr}}$, método padrão, com uso da radiação solar estimada pelo balanço entre ondas curtas e longas; HS, método de Hargreaves-Samani; e $\mathrm{PM}_{\mathrm{u} 2}$, método padrão, com uso da velocidade de vento constante $\left(2,0 \mathrm{~m} \mathrm{~s}^{-1}\right)$. 
Sentelhas et al. (2010) obtiveram valores de 0,79 a 1,12 mm por dia, para RMSE em estações do Canadá, com radiação solar estimada por meio do coeficiente Krs e das temperaturas máxima e mínima do ar. Os autores observaram que, na ausência de dados de velocidade do vento e umidade relativa, o uso de dados de estações próximas, dentro de uma mesma região homogênea, proporcionaram RMSE máximo de $0,60 \mathrm{~mm}$ por dia. De fato, o efeito da velocidade do vento na estimativa da ETo é relativamente pequeno, exceto para as zonas áridas e ventosas (Popova et al., 2006; Sentelhas et al., 2010).

$\mathrm{O}$ índice de ajustamento d de Willmott, que varia de 0 a 1 , representa o quanto os valores estimados se ajustam a valores medidos, ou seja, é uma medida de quão bem o modelo estima o afastamento dos dados da média observada (Willmott, 1981). De acordo com esse critério, o método $\mathrm{PM}_{\mathrm{u} 2}$ foi superior aos demais na estimativa da ETo no Espírito Santo, no Rio de Janeiro e em São Paulo, com desempenho classificado como ótimo (Figura $2 \mathrm{C}$ ). No entanto, em Minas Gerais, o método $\mathrm{PM}_{\mathrm{ea}}$ apresentou índice de ajustamento superior a 0,95, para todas as estações avaliadas, desempenho inferior ao obtido com os métodos $\mathrm{PM}_{\mathrm{u} 2}$ e $\mathrm{PM}_{\mathrm{p}}$ na estação de Mocambinho, cuja classificação pelo índice de ajustamento indicou desempenho "muito bom". É possível visualizar graficamente que o método de Turc, quanto a seu índice de ajustamento, mostrouse intermediário entre as estimativas mais e menos precisas, para a maioria das estações. Na Flórida, Thepadia \& Martinez (2012) verificaram predomínio de desvios negativos para o modelo de Turc, em relação à estimativa considerada padrão da FAO.

$\mathrm{O}$ modelo HS na comparação com o padrão $\left(\mathrm{PM}_{\mathrm{p}}\right.$ $\mathrm{x}$ HS) apresentou desempenho "ruim", para as estações de Arraial do Cabo e Parati, RJ. O modelo $\mathrm{PM}_{\mathrm{Krs}}$ também apresentou resultados ruins em Parati. Nas estações de Minas Gerais, registrou-se desempenho "ótimo" do modelo $\mathrm{PM}_{\mathrm{Krs}}$, e todos os modelos apresentaram desempenho "muito bom" ou "superior", exceto em Caldas, cuja classificação para HS apontou desempenho apenas "bom".

Conforme o exposto, foi possível estimar satisfatoriamente a ETo por abordagens simplificadas, desde a adoção de $\mathrm{u}_{2}$ constante até a estimativa de Rs a partir do fator Krs, Ra e da diferença entre as temperaturas máxima e mínima, bem como utilizar $\mathrm{UR}_{\text {med, }}, \mathrm{T}_{\text {máx }}$ e $\mathrm{T}_{\text {mín }}$ para estimar $\mathrm{e}_{\mathrm{a}}$ e compor a gama de parâmetros climáticos envolvidos na equação de Penmann-Monteith FAO-56 (Gocic \& Trajkovic, 2011).

\section{Conclusões}

1. O método padrão com uso da pressão de vapor estimada pelas temperaturas máxima e mínima e pela umidade relativa do ar $\left(\mathrm{PM}_{\mathrm{ea}}\right)$ é a melhor alternativa para estimar a evapotranspiração de referência (ETo), seguido pelo método padrão com uso da velocidade de vento constante $\left(\mathrm{PM}_{\mathrm{u} 2}\right)$, para Espírito Santo e Rio de Janeiro, e pelo método padrão com uso da radiação solar estimada pelo balanço entre ondas curtas e longas $\left(\mathrm{PM}_{\mathrm{Krs}}\right)$, para São Paulo e Minas Gerais.

2. A adoção de velocidade do vento constante a $2 \mathrm{~m} \mathrm{~s}^{-1}$ proporciona boa precisão e erros aceitáveis no Espírito Santo e no Rio de Janeiro, e consiste em alternativa adequada para estimativa da ETo com dados meteorológicos limitados.

3. O método de Hargreaves-Samani resulta nos maiores erros, com superestimativas em relação ao padrão $\mathrm{PM}_{\mathrm{p}}$, para a maioria das estações avaliadas.

4. $\mathrm{O}$ método $\mathrm{PM}_{\mathrm{ea}}$ é alternativa promissora para todas as estações de Minas Gerais, quando não se dispõe de medidas de temperatura do ponto de orvalho.

5. $\mathrm{O}$ método $\mathrm{PM}_{\mathrm{Krs}}$ tem desempenho estatístico satisfatório em regiões interioranas do País, mas não deve ser utilizado nas regiões litorâneas.

\section{Referências}

ALLEN, R.G.; PEREIRA, L.S.; HOWELL, T.A.; JENSEN, M.E. Evapotranspiration information reporting: I. Factors governing measurement accuracy. Agricultural Water Management, v.98, p.899-920, 2011a. DOI: 10.1016/j.agwat.2010.12.015.

ALLEN, R.G.; PEREIRA, L.S.; HOWELL, T.A.; JENSEN, M.E. Evapotranspiration information reporting: II. Recommended documentation. Agricultural Water Management, v.98, p.921-929, 2011b. DOI: 10.1016/j.agwat.2010.12.016.

ALLEN, R.G.; PEREIRA, L.S.; RAES, D.; SMITH, M. Crop evapotranspiration: guidelines for computing crop water requirements. Rome: FAO, 1998. (FAO irrigation and drainage paper, 56).

CAI, J.; LIU, Y.; LEI, T.; PEREIRA, L.S. Estimating reference evapotranspiration with the FAO Penman-Monteith equation using daily weather forecast messages. Agricultural and Forest Meteorology, v.145, p.22-35, 2007. DOI: 10.1016/j. agrformet.2007.04.012.

CARVALHO, D.F. de; OLIVEIRA, L.F.C. de. Planejamento e manejo da água na agricultura irrigada. Viçosa: Ed. UFV, 2012. 240 p. 
CARVALHO, D.F. de; SILVA, D.G. da; SOUZA, A.P. de; GOMES, D.P.; ROCHA, H.S. da. Coeficientes da equação de Angström-Prescott e sua influência na evapotranspiração de referência em Seropédica, RJ. Revista Brasileira de Engenharia Agrícola e Ambiental, v.15, p.838-844, 2011. DOI: 10.1590/ S1415-43662011000800011.

DOUGLAS, E.M.; JACOBS, J.M; SUMNER, D.M.; RAY, R.L. A comparison of models for estimating potential evapotranspiration for Florida land cover types. Journal of Hydrology, v.373, p.366-376, 2009. DOI: 10.1016/j.jhydrol.2009.04.029.

DROOGERS, P.; ALLEN, R.G. Estimating reference evapotranspiration under inaccurate data conditions. Irrigation and Drainage System, v.16, p.33-45, 2002. DOI: 10.1023/A:1015508322413.

FERNANDES, D.S.; HEINEMANN, A.B.; PAZ, R.L.F.; AMORIM, A. de O. Calibração regional e local da equação de Hargreaves para estimativa da evapotranspiração de referência. Revista Ciência Agronômica, v.43, p. 246-255, 2012. DOI: 10.1590/S1806-66902012000200006.

GOCIC, M.; TRAJKOVIC, S. Service-oriented approach for modeling and estimating reference evapotranspiration. Computers and Electronics in Agriculture, v.79, p.153-158, 2011. DOI: 10.1016/j.compag.2011.09.001.

HARGREAVES, G.H.; SAMANI, Z. Reference crop evapotranspiration from ambient air temperature. Chicago: American Society of Agricultural and Biological Engineers Meeteng, 1985. (Paper 85-2517).

INSTITUTO BRASILEIRO DE GEOGRAFIA E ESTATÍSTICA. Levantamento sistemático da produção agrícola: pesquisa mensal de previsão e acompanhamento das safras agrícolas no ano civil: abril de 2013. 2013. Disponível em: <http://www.ibge.gov. br/home/estatistica/indicadores/agropecuaria/lspa/lspa_201304. pdf $>$. Acesso em: 24 maio 2013.

KUMAR, M.; BANDYOPADHYAY, A.; RAGHUWANSHI, N.S.; SINGH, R. Comparative study of conventional and artificial neural network-based ETo estimation models. Irrigation Science, v.26, p.531-545, 2008. DOI: 10.1007/S00271-008-0114-3.

LANDERAS, G.; ORTIZ-BARREDO, A.; LÓPEZ, J.J. Comparison of artificial neural network models and empirical and semi-empirical equations for daily reference evapotranspiration estimation in the Basque Country (Northern Spain). Agricultural Water Management, v.95, p.553-565, 2008. DOI: 10.1016/j. agwat.2007.12.011.

MARTÍ, P.; ROYUELA, A.; MANZANO, J.; PALAU-SALVADOR, G. Generalization of ETo ANN models through data supplanting. Journal of Irrigation and Drainage Engineering, v.136, p.161-174, 2010. DOI: 10.1061/(ASCE)IR.1943-4774.0000152.

MARTINEZ, C.J.; THEPADIA, M. Estimating reference evapotranspiration with minimum data in Florida. Journal of
Irrigation and Drainage Engineering, v.136, p.494-501, 2010. DOI: 10.1061/(ASCE)IR.1943-4774.0000214.

POPOVA, Z.; KERCHEVA, M.; PEREIRA, L.S. Validation of the FAO methodology for computing $\mathrm{ET}_{\mathrm{o}}$ with limited data. Application to South Bulgaria. Irrigation and Drainage, v.55, p.201-215, 2006. DOI: 10.1002/ird.228.

RAZIEI, T; PEREIRA, L.S. Estimation of $\mathrm{ET}_{0}$ with Hargreaves-Samani and FAO-PM temperature methods for a wide range of climates in Iran. Agricultural Water Management, v.121, p.1-18, 2013. DOI: 10.1016/j.agwat.2012.12.019.

ROCHA, É. da J.T.; EVANGELISTA, S.R.M.; FUCK JÚNIOR, S.C. de F.; GONDIM, R.S. Estimativa da $\mathrm{ET}_{\mathrm{o}}$ pelo modelo Penman-Monteith FAO com dados mínimos integrada a um Sistema de Informação Geográfica. Revista Ciência Agronômica, v.42, p.75-83, 2011. DOI: 10.1590/ S1806-66902011000100010.

ROJAS, J.P.; SHEFFIELD, R.E. Evaluation of daily reference evapotranspiration methods as compared with the ASCE-EWRI Penman-Monteith equation using limited weather data in northeast Louisiana. Journal of Irrigation and Drainage Engineering, v.139, p.285-292, 2013. DOI: 10.1061/(ASCE) IR.1943-4774.0000523.

SENTELHAS, P.C.; GILLESPIE, T.J.; SANTOS, E.A. Evaluation of FAO Penman-Monteith and alternative methods for estimating reference evapotranspiration with missing data in Southern Ontario, Canada. Agricultural Water Management, v.97, p.635-644, 2010. DOI: 10.1016/j.agwat.2009.12.001.

SOUZA,A.P. de; CARVALHO, D.F. de; SILVA, L.B. da;ALMEIDA, F.T. de; ROCHA, H.S. da. Estimativas da evapotranspiração de referência em diferentes condições de nebulosidade. Pesquisa Agropecuária Brasileira, v.46, p.219-228, 2011. DOI: 10.1590/ S0100-204X2011000300001.

STONE, R.J. Improved statistical procedure for the evaluation of solar radiation estimation models. Solar Energy, v.51, p.289-291, 1993. DOI: 10.1016/0038-092X(93)90124-7.

THEPADIA, M.; MARTINEZ, C.J. Regional calibration of solar radiation and reference evapotranspiration estimates with minimal data in Florida. Journal of Irrigation and Drainage Engineering, v.138, p.111-119, 2012. DOI: 10.1061/(ASCE) IR.1943-4774.0000394.

TODOROVIC, M.; KARIC, B.; PEREIRA, L.S. Reference evapotranspiration estimate with limited weather data across a range of Mediterranean climates. Journal of Hydrology, v.481, p.166-176, 2013. DOI: 10.1016/j.jhydrol.2012.12.034.

TRAJKOVIC, S.; KOLAKOVIC, S. Estimating reference evapotranspiration using limited weather data. Journal of Irrigation and Drainage Engineering, v.135, p.443-449, 2009. DOI: 10.1061/(ASCE)IR.1943-4774.0000094.

WILlMOTT, C.J. On the validation of models. Physical Geography, v.2, p.184-194, 1981.

Recebido em 15 de maio de 2014 e aprovado em 4 de novembro de 2014 